\title{
LHCb status and early physics prospects
}

\author{
M. Pepe Altarelli $\left.{ }^{*}\right)$ on behalf of the LHCb Collaboration \\ CERN, PH Department - CH-1211 Geneve 23, Switzerland
}

(ricevuto il 10 Novembre 2009; pubblicato online il 18 Gennaio 2010)

\begin{abstract}
Summary. - LHCb is a dedicated detector for b and c physics at the LHC. I will present a concise review of the detector design and expected performance together with some first results on the commissioning of the different sub-systems based on cosmic data and particle beams delivered by the LHC during the summer of 2008. The experiment is ready to exploit first data expected from the LHC. An integrated luminosity of $\sim 0.3 \mathrm{fb}^{-1}$, which should be collected during the first year of physics running, will already allow $\mathrm{LHCb}$ to perform a number of very significant mesurements with the potential of revealing New Physics effects, such as the measurement of the $\mathrm{B}_{\mathrm{s}}$ mixing phase $\phi_{\mathrm{J} / \psi \phi}$, or the search of the decay $\mathrm{B}_{\mathrm{s}}^{0} \rightarrow$ $\mu^{+} \mu^{-}$beyond the limit set by CDF and D0.

PACS 12.15.Ff - Quark and lepton masses and mixing.

PACS 12.15. Hh - Determination of Kobayashi-Maskawa matrix elements.

PACS 12.60.-i - Models beyond the standard model.

PACS 13.20.He - Decays of bottom mesons.
\end{abstract}

\section{1. - Introduction}

$\mathrm{LHCb}$ is a dedicated b- and c-physics precision experiment at the LHC that will search for New Physics (NP) beyond the Standard Model (SM) through the study of very rare decays of charm and beauty-flavoured hadrons and precision measurements of $C P$-violating observables. In the last decade, experiments at $\mathrm{B}$ factories have confirmed that the mechanism proposed by Kobayashi and Maskawa is the major source of $C P$ violation observed so far. The SM description of flavour-changing processes has been confirmed in the $\mathrm{b} \rightarrow \mathrm{d}$ transition at the level of 10-20\% accuracy. However, NP effects can still be large in $\mathrm{b} \rightarrow \mathrm{s}$ transitions, modifying the $\mathrm{B}_{\mathrm{s}}$ mixing phase $\phi_{\mathrm{J} / \psi \phi}$, measured from $\mathrm{B}_{\mathrm{s}}^{0} \rightarrow \mathrm{J} / \psi \phi$ decays, or in channels dominated by other loop diagrams, such as the very rare decay $\mathrm{B}_{\mathrm{s}}^{0} \rightarrow \mu^{+} \mu^{-}$, or in $\mathrm{B}_{\mathrm{s}}^{0} \rightarrow \phi \phi$. Therefore, the challenge of the future $\mathrm{b}$ experiments is to widen the range of measured decays, reaching channels that are strongly

(*) E-mail: monica.pepe.altarelli@cern.ch

(C) Società Italiana di Fisica / INFN - Laboratori Nazionali di Frascati 
suppressed in the SM and, more generally, to improve the precision of the measurements to achieve the necessary sensitivity to NP effects in loops. LHCb will extend the b-physics results from the $\mathrm{B}$ factories by studying decays of heavier b hadrons, such as $\mathrm{B}_{\mathrm{s}}$ or $\mathrm{B}_{\mathrm{c}}$, which will be copiously produced at the LHC. It will complement the direct search of NP at the LHC by providing important information on the NP flavour structure through a dedicated detector, optimized for this kind of physics.

\section{2. - b physics at the LHC: environment, background, general trigger issues}

The LHC will be the world's most intense source of b-hadrons. In proton-proton collisions at $\sqrt{s}=14 \mathrm{TeV}$, the $\mathrm{b} \overline{\mathrm{b}}$ cross-section is expected to be $\sim 500 \mu \mathrm{b}$ producing $10^{12} \mathrm{~b} \overline{\mathrm{b}}$ pairs in a standard $\left(10^{7} \mathrm{~s}\right)$ year of running at the LHCb operational luminosity of $2 \times 10^{32} \mathrm{~cm}^{-2} \mathrm{~s}^{-1}$. As in the case of the Tevatron, a complete spectrum of b-hadrons will be available, including $B_{\mathrm{s}}, B_{\mathrm{c}}$ mesons and baryons such as $\Lambda_{\mathrm{b}}$. However, less than $1 \%$ of all inelastic events contain b quarks, hence triggering is a critical issue.

At the nominal LHC design luminosity of $10^{34} \mathrm{~cm}^{-2} \mathrm{~s}^{-1}$, multiple p-p collisions within the same bunch crossing (so-called pile-up) would significantly complicate the b-production and decay-vertex reconstruction. For this reason the luminosity at $\mathrm{LHCb}$ will be locally controlled by appropriately focusing the beam to yield $\mathcal{L}=2-5 \times$ $10^{32} \mathrm{~cm}^{-2} \mathrm{~s}^{-1}$, at which the majority of the events have a single p-p interaction. This matches well with the expected LHC conditions during the start-up phase. Furthermore, running at relatively low luminosity reduces the detector occupancy of the tracking systems and limits radiation damage effects.

The dominant production mechanism at the LHC is through gluon-gluon fusion in which the momenta of the incoming partons are strongly asymmetric in the p-p centreof-mass frame. As a consequence, the $b \bar{b}$ pair is boosted along the direction of the higher momentum gluon, and both b-hadrons are produced in the same forward (or backward) direction in the p-p centre-of-mass frame. The detector is therefore designed as a single arm forward spectrometer covering the pseudorapidity range $1.9<\eta<4.9$, which ensures a high geometric efficiency for detecting all the decay particles from one b-hadron together with the decay particles from the accompanying $\bar{b}$-hadron to be used as a flavour tag. A modification to the LHC optics, displacing the interaction point by $11.25 \mathrm{~m}$ from the centre, has permitted maximum use to be made of the existing cavern by freeing $19.7 \mathrm{~m}$ for the $\mathrm{LHCb}$ detector components.

A detector design based on a forward spectrometer offers further advantages: b-hadrons are expected to have a hard momentum spectrum in the forward region; their average momentum is $\sim 80 \mathrm{GeV} / c$, corresponding to approximately $7 \mathrm{~mm}$ mean decay distance, which facilitates the separation between primary and decay vertices. This property, coupled to the excellent vertex resolution capabilities, allows proper time to be measured with a resolution of $\sim 40 \mathrm{fs}$, which is crucial for studying $C P$ violation and oscillations with $\mathrm{B}_{\mathrm{s}}$-mesons, because of their high oscillation frequency. Furthermore, the forward, open geometry allows the vertex detector to be positioned very close to the beams and facilitates detector installation and maintenance. In particular, the silicon detector sensors, housed, like Roman pots, in a secondary vacuum, are split in two halves that are retracted by $\sim 30 \mathrm{~mm}$ from the interaction region before the LHC ring is filled, in order to allow for beam excursions during injection and ramping. They are then positioned within $\sim 8 \mathrm{~mm}$ from the interaction region after stable beam conditions have been obtained. 


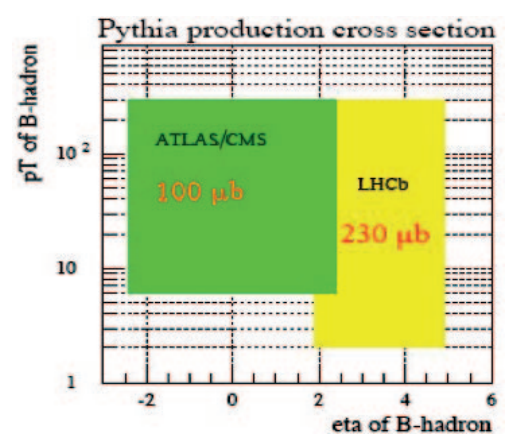

Fig. 1. - b-hadron transverse momentum as a function of the pseudorapidity $\eta$, showing the $\left(\eta, p_{\mathrm{T}}\right)$ region covered by ATLAS and CMS, compared to that covered by LHCb.

Figure 1 illustrates the $\mathrm{LHCb}$ acceptance in the plane $\left(\eta, p_{\mathrm{T}}\right)$ of the b-hadrons in comparison to that of ATLAS and CMS: ATLAS and CMS cover a pseudorapidity range of $|\eta|<2.5$ and rely on high- $p_{\mathrm{T}}$ lepton triggers. LHCb relies on much lower $p_{\mathrm{T}}$ triggers, which are efficient also for purely hadronic decays. Most of the ATLAS and CMS b-physics programme will be pursued during the first few years of operation, for luminosities of order $10^{33} \mathrm{~cm}^{-2} \mathrm{~s}^{-1}$. Once LHC reaches its design luminosity, b physics will become exceedingly difficult for ATLAS and CMS due to the large pile-up (20 interactions per bunch crossing, on average), except for very few specific channels characterized by a simple signature, like $\mathrm{B}_{\mathrm{s}}^{0} \rightarrow \mu^{+} \mu^{-}$.

\section{3. - Detector description and performance}

The key features of the LHCb detector are:

- A versatile trigger scheme efficient for both leptonic and hadronic final states, which is able to cope with a variety of modes with small branching fractions;

- Excellent vertex and proper time resolution;

- Precise particle identification (ID), specifically for hadron $(\pi / \mathrm{K})$ separation;

- Precise invariant mass reconstruction to efficiently reject background due to random combinations of tracks. This implies a high momentum resolution.

A schematic layout is shown in fig. 2. It consists of a vertex locator (VELO), a charge particle tracking system with a large aperture dipole magnet, aerogel and gas Ring Imaging Cherenkov counters (RICH), electromagnetic (ECAL) and hadronic (HCAL) calorimeters and a muon system. In the following, the most salient features of the $\mathrm{LHCb}$ detector are described in more detail. A much more complete description of the detector characteristics can be found in $[1,2]$.

3•1. Trigger. - One of the most critical elements of $\mathrm{LHCb}$ is the trigger system. At the chosen LHCb nominal luminosity, taking into account the LHC bunch crossing structure, the rate of events with at least two particles in the $\mathrm{LHCb}$ acceptance is $\sim 10 \mathrm{MHz}$ (instead of the nominal $40 \mathrm{MHz}$ LHC crossing rate). The rate of events containing b-quarks is $\sim 100 \mathrm{kHz}$, while the rate of events containing c quarks is much larger $(\sim 600 \mathrm{kHz})$. However, the rate of interesting events is just a very small fraction of the total rate 


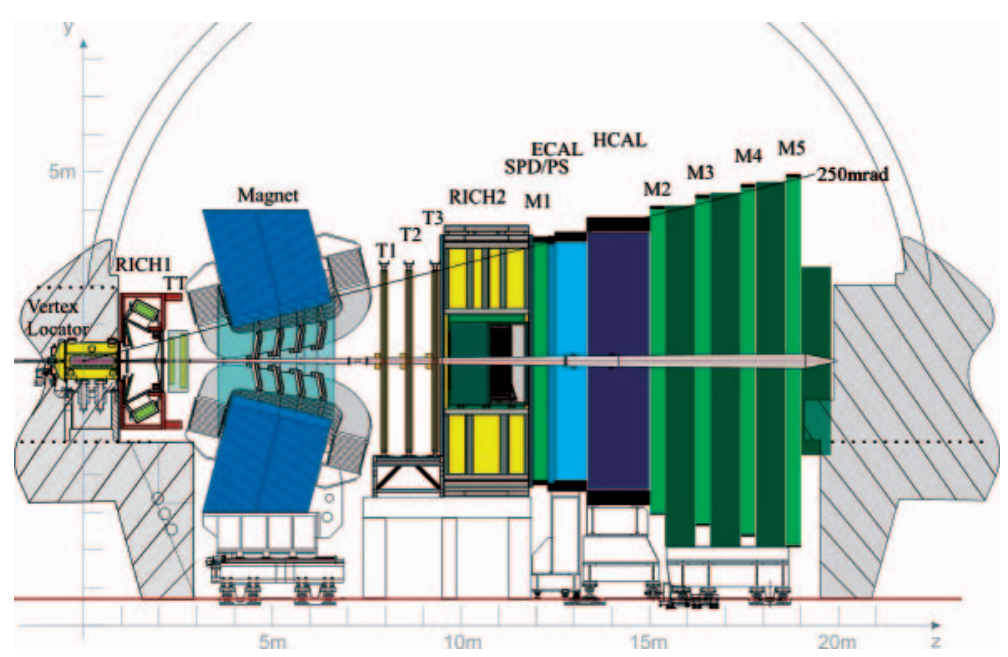

Fig. 2. - Side view of the LHCb detector showing the Vertex Locator (VELO), the dipole magnet, the two RICH detectors, the four tracking stations TT and T1-T3, the Scintillating Pad Detector (SPD), Preshower (PS), Electromagnetic (ECAL) and Hadronic (HCAL) calorimeters, and the five muon stations M1-M5.

$(\sim \mathrm{Hz})$, due to the combined effect of branching fraction and detector acceptance, hence the need for a highly selective and efficient trigger.

The LHCb trigger exploits the fact that b-hadrons are long-lived, resulting in well-separated primary and secondary vertices, and have a relatively large mass, resulting in decay products with large $p_{\mathrm{T}}$. It consists of two levels: Level0 (L0) and High Level Trigger (HLT). L0, implemented on custom electronics boards, is designed to reduce the input rate to $1 \mathrm{MHz}$ at a fixed latency of $4 \mu \mathrm{s}$. At this rate, events are sent to a computer farm with up to $\sim 2000$ multiprocessor boxes where several HLT software algorithms are executed. The HLT, which has access to the full detector information, reduces the rate from $1 \mathrm{MHz}$ to $\sim 2 \mathrm{kHz}$.

L0, based on calorimeter and muon chamber information, selects muons, electrons, photons or hadrons above a given $p_{\mathrm{T}}$ or $E_{\mathrm{T}}$ threshold, typically in the range 1 to $4 \mathrm{GeV}$. The L0 hadron trigger occupies most of the bandwidth $(700 \mathrm{kHz})$ and is unique within the LHC experiments. The muon triggers (single and double) select $\sim 200 \mathrm{kHz}$, while the rest of the bandwidth is due to the electromagnetic calorimeter triggers. Typically, the L0 efficiency is $\sim 50 \%$ for hadronic channels, $\sim 90 \%$ for muon channels and $\sim 70 \%$ for radiative channels, normalized to offline selected events.

The HLT algorithms are designed to be simple, to minimize systematic uncertainties, and fast. This is realized by reconstructing for each trigger only a few tracks, which are used for the final decision. The HLT comprises several paths (alleys) to confirm and progressively refine the $\mathrm{L} 0$ decision, followed by inclusive and exclusive selections. The choice of the alley depends on the L0 decision. The average execution time is few ms, which matches with the expected size of the CPU farm. The total trigger rate after the HLT is $\sim 2 \mathrm{kHz}$, a relatively high rate that also includes calibration samples to be used to understand the detector performance.

3.2. VELO and tracking system. - The LHCb tracking system consists of a warm dipole magnet, which generates a magnetic field integral of $\sim 4 \mathrm{Tm}$, four tracking stations 
and the VELO. The first tracking station located upstream of the magnet consists of four layers of silicon strip detectors. The remaining three stations downstream of the magnet are each constructed from four double layers of straw tubes in the outer region, covering most $(\sim 98 \%)$ of the tracker area, and silicon strips in the area closer to the beam pipe $(\sim 2 \%)$. However, $\sim 20 \%$ of the charged particles traversing the detector go through the silicon inner tracker, due to the forward-peaked multiplicity distribution. The expected momentum resolution increases from $\delta p / p \sim 0.35 \%$ for low-momentum tracks to $0.55 \%$ at the upper end of the momentum spectrum. This translates into an invariant mass resolution of $\delta M \sim 20 \mathrm{MeV} / c^{2}$ for $\mathrm{B}_{\mathrm{s}}$ decays into two charged tracks, such as $\mathrm{B}_{\mathrm{s}}^{0} \rightarrow \mu^{+} \mu^{-}$, substantially better than in the general-purpose detectors at LHC.

The VELO consists of 21 stations, each made of two silicon half-disks, which measure the radial and azimuthal coordinates. The VELO has the unique feature of being located at a very close distance from the beam line $(0.8 \mathrm{~cm})$, inside a vacuum vessel, separated from the beam vacuum by a thin aluminum foil. This allows an impressive vertex resolution to be achieved, translating, for instance, in a proper time resolution of $\sim 36 \mathrm{fs}$ for the decay $\mathrm{B}_{\mathrm{s}}^{0} \rightarrow \mathrm{J} / \psi \phi$, i.e. a factor of ten smaller than the $\mathrm{B}_{\mathrm{s}}$ oscillation period and a factor of two better than in the general-purpose detectors. The resolution on the impact parameter can be parameterized as $\delta I P \sim 14 \mu \mathrm{m}+35 \mu \mathrm{m} / p_{\mathrm{T}}$.

3.3. Particle identification. - Particle identification is provided by the two RICH detectors and the calorimeter and muon systems.

The RICH system is one of the crucial components of the $\mathrm{LHCb}$ detector. The first $\mathrm{RICH}$, located upstream of the magnet, employs two radiators, $\mathrm{C}_{4} \mathrm{H}_{10}$ gas and aerogel, ensuring a good separation in the momentum range from 2 to $60 \mathrm{GeV} / c$. A second $\mathrm{RICH}$ in front of the calorimeters, uses a $\mathrm{CF}_{4}$ gas radiator and extends the momentum coverage up to $\sim 100 \mathrm{GeV} / c$. The calorimeter system comprises a pre-shower detector consisting of 2.5 radiation length lead sheet sandwiched between two scintillator plates, a 25 radiation length lead-scintillator electromagnetic calorimeter of the shashlik type and a 5.6 interaction length iron-scintillator hadron calorimeter. The muon detector consists of five muon stations equipped with multiwire proportional chambers, with the exception of the centre of the first station, which uses triple-GEM detectors.

Electrons, photons and $\pi^{0} \mathrm{~s}$ are identified using the calorimeter system. The average electron identification efficiency extracted from $\mathrm{J} / \psi \rightarrow \mathrm{e}^{+} \mathrm{e}^{-}$decays is $\sim 95 \%$ for a pion misidentification rate of $\sim 0.7 \%$. Muons are identified using the muon detector with an average efficiency in the acceptance extracted from $\mathrm{J} / \psi \rightarrow \mu \mu$ decays of $\sim 93 \%$ for a pion misidentification rate of $\sim 1 \%$. The RICH system provides good particle identification over the entire momentum range. The average efficiency for kaon identification for momenta between 2 and $100 \mathrm{GeV} / c$ is $\epsilon(\mathrm{K} \rightarrow \mathrm{K}) \sim 95 \%$, with a corresponding average pion misidentification rate $\epsilon(\pi \rightarrow \mathrm{K}) \sim 5 \%$.

\section{4. - Commissioning}

During the summer of 2008 the commissioning activities converged into a fully operational detector. All sub-detectors were included under central control and data taking was extensively exercised. Although the geometry of the LHCb detector is not well suited for cosmic runs (the rate of "horizontal" cosmic events is well below $1 \mathrm{~Hz}$ ), over one million cosmic events were recorded using muon and calorimeter cosmic triggers. These cosmic data have proved to be extremely useful to perform a coarse initial geometrical and time alignment of the larger detectors. As an example, fig. 3 shows the distributions 


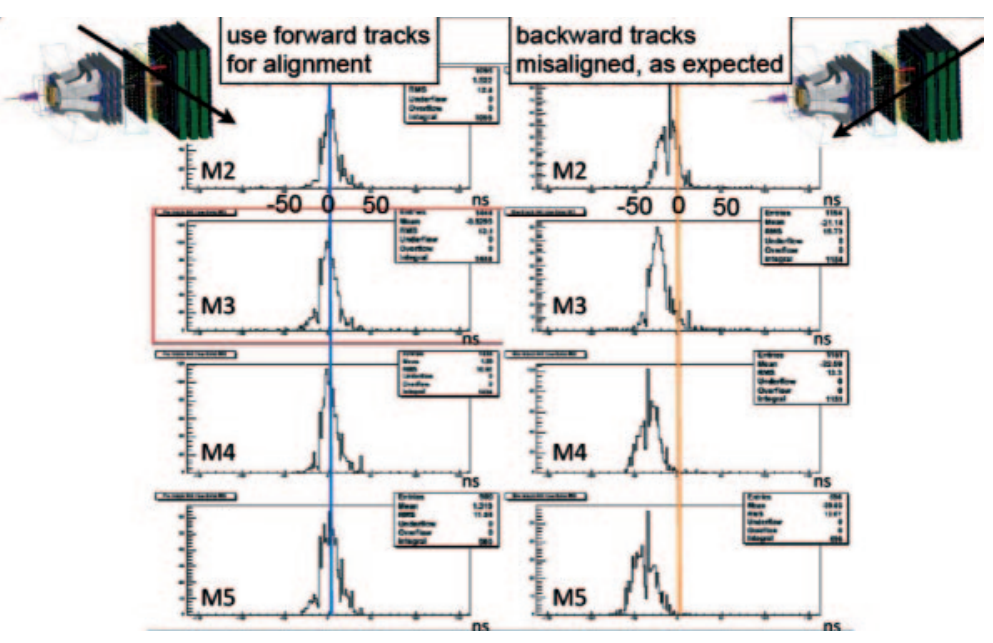

Fig. 3. - Readout time distribution with respect to reference time (in ns) for forward and backward tracks in four muon stations (M2-M5).

of the readout time, optimized for forward tracks, of four muon stations. The readout time is measured with respect to the trigger time as provided by the scintillator pad detector of the calorimeter system. The distributions are nicely centred at zero only for the forward tracks.

At the end of August 2008, the machine carried out several tests of the transfer line ("synchronization tests"). A beam of $450 \mathrm{GeV}$ protons extracted from the SPS was injected into the LHC and dumped on an injection line beam stopper ("TED") located approximately $340 \mathrm{~m}$ downstream of LHCb. This produced a large flux of nearly parallel particles that hit the detector from the back, i.e. from the muon stations towards the VELO. These data were particularly useful for the time and position alignment of those sub-detectors (IT, TT and VELO) that could not make use of cosmic events, as they are either too small or too distant from the detectors providing the trigger. The initial alignment with reconstructed tracks indicated no major problems and a resolution in the expected range.

As an example, for the VELO the detector displacement was measured to be less than $\sim 10 \mu \mathrm{m}$ with respect to the survey information. The achieved resolution was measured to match the binary resolution $\left(=\frac{\text { pitch }}{\sqrt{12}}\right)$, which is consistent with expectation, given that the large majority $(\sim 90 \%)$ of the used clusters are one-strip clusters. Improvements to the resolution are expected with an optimized tuning of the signal processing algorithms and of the readout time alignment. Moreover, the resolution is expected to be better for tracks at angles around $140 \mathrm{mrad}$, for which the charge sharing between adjacent strips is optimal.

\section{5. - Early physics at $\mathrm{LHCb}$}

The objective of the very first running phase is to complete the commissioning of the sub-detectors and of the trigger. Large minimum bias data samples $\left(\sim 10^{8}\right.$ per day at $2 \mathrm{kHz}$ output rate) will be collected as soon as the LHC delivers p-p collisions using a simple interaction trigger based on total energy in the calorimeters. These data will provide a high-statistics and high-purity $\mathrm{V}^{0}$ sample $\left(\mathrm{K}_{\mathrm{S}}^{0}, \Lambda, \bar{\Lambda}\right)$, which can be used to probe 


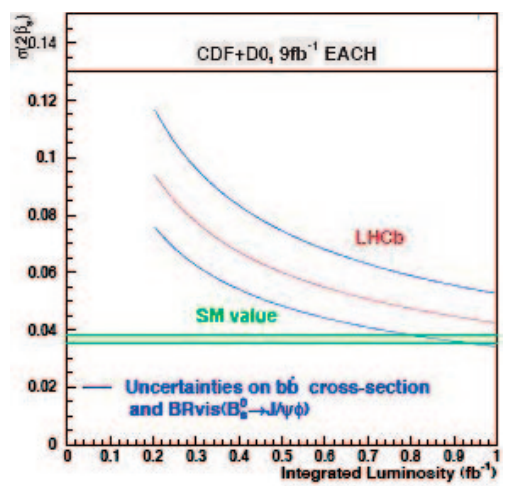

Fig. 4. - (Colour online) Statistical uncertainty on $\phi_{\mathrm{J} / \psi \phi} v s$. the integrated luminosity. The blue lines show the uncertainties coming from the $b \bar{b}$ cross-section and the visible branching ratio on $\mathrm{B}_{\mathrm{s}}^{0} \rightarrow \mathrm{J} / \psi \phi$. The green band is the $\mathrm{SM}$ value. The Tevatron line is the combined CDF/D0 uncertainty in 2008 scaled to $18 \mathrm{fb}^{-1}$, as expected by the end of run 2 .

the hadronization process in a rapidity range complementary to that of the other LHC detectors and in which phenomenological models tuned to Tevatron data show significant differences when extrapolated to LHC energies. Measurements will include differential cross-sections and production ratios for different strange particles as a function of rapidity and transverse momentum.

A simple, lifetime-unbiased muon trigger, requiring $p_{\mathrm{T}}>1 \mathrm{GeV} / c$ at L0, will allow us to collect a large, clean sample of $\mathrm{J} / \psi \rightarrow \mu \mu$ decays. With an integrated luminosity of $5 \mathrm{pb}^{-1}, \sim 3 \times 10^{6}$ events are expected with a $\mathrm{S} / \mathrm{B}$ of $\sim 4$ at $\sqrt{s}=8 \mathrm{TeV}$, which may be the initial LHC centre-of-mass energy. This sample can be used to extract both the prompt $\mathrm{J} / \psi$ and $\mathrm{b} \rightarrow \mathrm{J} / \psi$ production cross-sections in a region not accessible to other collider experiments. Other charmonia related measurements will also be performed, such as that of the $\mathrm{J} / \psi$ polarization or of the production of the exotic $\mathrm{X}, \mathrm{Y}$ and $\mathrm{Z}$ charmonia states observed in recent years.

An integrated luminosity of $0.2-0.3 \mathrm{fb}^{-1}$, which should hopefully be collected during the first year of physics running, will already allow LHCb to realize a number of very significant b-physics measurements, with the potential of revealing NP effects, such as the measurement of the $\mathrm{B}_{\mathrm{s}}$ mixing phase $\phi_{\mathrm{J} / \psi \phi}$, the search of the decay $\mathrm{B}_{\mathrm{s}}^{0} \rightarrow \mu^{+} \mu^{-}$ beyond the limit set by CDF and D0, or the study of the decay $\mathrm{B}_{\mathrm{d}} \rightarrow \mathrm{K}^{* 0} \mu^{+} \mu^{-}$(for this last decay, with $0.2 \mathrm{fb}^{-1} \mathrm{LHCb}$ should be able to collect $\sim 700$ events, which is a larger sample than at all existing facilities combined).

A flavour tagged, angular analysis of the decay $\mathrm{B}_{\mathrm{s}}^{0} \rightarrow \mathrm{J} / \psi \phi$ allows the determination of a $C P$-violating phase $\phi_{\mathrm{J} / \psi \phi}$. In the $\mathrm{SM}$ this phase is predicted to be $-2 \beta_{\mathrm{s}} \simeq-0.04$, where $\beta_{\mathrm{s}}$ is the smaller angle of the "b-s unitarity triangle". However NP could significantly modify this prediction, if new particles contribute to the $\mathrm{B}_{\mathrm{s}}^{0}-\overline{\mathrm{B}_{\mathrm{s}}^{0}}$ box diagram. In fact, the $\mathrm{CDF}$ and D0 Collaborations [3,4] have reported a first measurement of the $\mathrm{B}_{\mathrm{s}}$ mixing phase. Their combined result deviates from the SM prediction by $\sim 2.2 \sigma$ with a central value for $2 \beta_{\mathrm{s}}$ as large as 0.77 . LHCb has the capability to significantly improve the existing experimental knowledge of this phase thanks to the large signal yield $(\sim 12 \mathrm{k}$ events for $\left.0.2 \mathrm{fb}^{-1}\right)$, the excellent proper time resolution to resolve fast $\mathrm{B}_{\mathrm{s}}$ oscillations ( $\sim 40 \mathrm{fs}$ ), the good flavour tagging $(\sim 6 \%)$, and the good control of the proper time and angular acceptance. Figure 4 shows the statistical uncertainty on the phase $\phi_{\mathrm{J} / \psi \phi}$ 


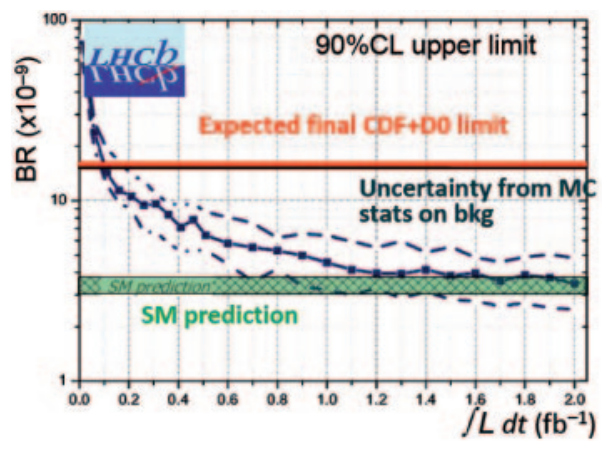

Fig. 5. - (Colour online) Expected 90\% CL upper limit of $\mathrm{BR}\left(\mathrm{B}_{\mathrm{s}}^{0} \rightarrow \mu^{+} \mu^{-}\right)$in the absence of signal as a function of the integrated luminosity. The green band is the SM value. The Tevatron line is the combined CDF/D0 uncertainty in 2008 scaled to $18 \mathrm{fb}^{-1}$, as expected by the end of run 2 .

as a function of the integrated luminosity. As one can see, already with $0.3 \mathrm{fb}^{-1}, \mathrm{LHCb}$ should be able to improve on the expected Tevatron limit.

The decay $\mathrm{B}_{\mathrm{S}}^{0} \rightarrow \mu^{+} \mu^{-}$has been identified as another very interesting potential constraint on the parameter space of models for physics beyond the SM. The BR for this decay is computed to be very small in the $\mathrm{SM}: \mathrm{BR}\left(\mathrm{B}_{\mathrm{s}}^{0} \rightarrow \mu^{+} \mu^{-}\right)=(3.35 \pm 0.32) \times 10^{-9}[5]$, but could be enhanced in certain NP scenarios. For example, in the MSSM, this branching ratio is known to increase as the sixth power of $\tan \beta=\nu_{\mathrm{u}} / \nu_{\mathrm{d}}$, the ratio of the two vacuum expectation values. Any improvement to this limit is therefore particularly important for models with large $\tan \beta$. The upper limit to the $\mathrm{B}_{\mathrm{s}}^{0} \rightarrow \mu^{+} \mu^{-}$branching ratio measured at the Tevatron is $\mathrm{BR}\left(\mathrm{B}_{\mathrm{s}}^{0} \rightarrow \mu^{+} \mu^{-}\right)<4.7 \times 10^{-8}$ at $90 \% \mathrm{CL}[6,7]$. For this measurement, $\mathrm{LHCb}$ has developed an analysis based on the use of control channels to minimize the dependence on MC simulation. The main issue for this analysis is the rejection of the background, largely dominated by random combinations of two muons originating from two distinct $b$ decays. This background can be kept under control by exploiting the excellent $\mathrm{LHCb}$ vertexing capabilities, and mass resolution. Figure 5 shows, as a function of the integrated luminosity, the BR value excluded at 90\% CL. Already with $\sim 0.2 \mathrm{fb}^{-1}$ LHCb should improve on the expected Tevatron limit, while $\mathrm{a} \sim 3 \sigma$ observation will require an integrated luminosity of $3 \mathrm{fb}^{-1}$, assuming the $\mathrm{SM}$ value.

In subsequent years, the experiment will develop its full physics programme, and plans are to accumulate an integrated luminosity of $\sim 10 \mathrm{fb}^{-1}$. Such a data sample will, for example, allow LHCb to improve the error on the CKM angle $\gamma$ by a factor of $\sim$ five, and probe NP in rare B-meson decays with electroweak, radiative and hadronic penguin modes.

\section{6. - Conclusions}

The large $\mathrm{b} \overline{\mathrm{b}}$ production cross-section at the LHC provides a unique opportunity to study in detail $C P$ violation and rare b decays with the $\mathrm{LHCb}$ detector. In particular, production of $\mathrm{B}_{\mathrm{s}}$ mesons could play a crucial role in disentangling effects originating from NP and a few observables sensitive to NP should already be accessible at the end of the 1st year of data taking. During the last year LHCb was fully installed and commissioned 
using cosmic events and first LHC-induced tracks. At this point we are all eagerly waiting to exploit first data expected from the LHC in 2009/2010.

I would like to thank the Organizers of Les Rencontres for their kind invitation and my LHCb colleagues for providing the material discussed in this article, in particular TATSUYA NAKADA for taking the time to read this manuscript.

\section{REFERENCES}

[1] LHCb Collaboration, LHCb Reoptimized Detector, Design and Performance, LHCC2003-030 (2003).

[2] LHCb Collaboration, The LHCb Detector at the LHC, JINST, 3 (2008) S08005.

[3] CDF Collaboration, First Flavor-Tagged determination of Bounds on Mixing-Induced $C P$ Violation in $\mathrm{B}_{\mathrm{s}}^{0} \rightarrow \mathrm{J} / \psi \phi$ Decays, Phys. Rev. Lett., 100 (2008) 161802.

[4] D0 Collaboration, Measurement of $\mathrm{B}_{\mathrm{s}}$ mixing parameters from the flavor-tagged decay $\mathrm{B}_{\mathrm{s}}^{0} \rightarrow \mathrm{J} / \psi \phi$, arXiv:0802.2255v1 [hep-ex] 2008.

[5] Blanke M., Buras. A., Guadagnoli D. and Tarantino C., arXiv:hep-ph/0604057v5 2006.

[6] CDF Collaboration, Phys. Rev. A, 100 (2008) 101802.

[7] D0 Collaboration, D0 note 5344-CONF (2007). 